Abstract 1808 Table 1 CRP results

\begin{tabular}{lcccc}
\hline Group & $\mathbf{n}=$ & CRP & Result & t-test \\
\hline PA v. NO-PA & 82 vs. 22 & $\# 1$ & $0.5+1.2$ vs. $0.4+0.4$ & $p=0.33$ \\
PA v. NO-PA & 78 vs. 14 & $\# 2$ & $1.0+1.3$ vs. $0.4+0.3$ & $p<0.01$ \\
PA v. NO-PA & 61 vs. 6 & $\# 3$ & $1.1+1.7$ vs. $0.2+0.0$ & $p<0.01$ \\
\hline
\end{tabular}

Conclusions CRP significantly increased in PA v. NO-PA supporting prior reports. We theorize inflammation is caused by peroxidation of polyunsaturated fatty acids in PA. A clinical trial is needed that studies cytologic and biochemical findings in tracheal aspirates after PA therapy and this will alleviate safety concerns.

\section{REDUCED DURATION OF CPAP IN PRETERM BABIES RECEIVING KANGAROO CARE WITHIN AN HOUR OF BIRTH - RANDOMIZED TRIAL}

doi:10.1136/archdischild-2012-302724.1809

1,2SM Nimbalkar, 'R Tandon, 'NS Chaudhary. 'Department of Pediatrics, Pramukhswami Medical College; ${ }^{2}$ Central Research Services, Charutar Arogya Mandal, Anand, India

Background and Aims Kangaroo Mother Care (KMC) is conventionally initiated in a baby who is otherwise stable but may still be on intravenous fluids, tube feeding and/or oxygen. We hypothesized that initiating KMC earlier will reduce the requirement for oxygen and the duration of respiratory support if Continuous Positive Airway Pressure (CPAP) was started along with Kangaroo care immediately after delivery in premature babies with respiratory distress. Methods Prospective randomized controlled trial in a total of 16 preterm neonates with respiratory distress syndrome who were assigned to CPAP (Fischer Paykel Bubble CPAP generator with short bi-nasal prongs) with or without KMC within 1 hour of starting CPAP. Primary outcome was requirement of oxygen and mechanical ventilation. Secondary outcome was early initiation of feed, apnoeic episodes and number of days for achieving maximum feed.

Results 13 babies were randomised into intervention group and 12 in control group. The mean weight was $1.51 \mathrm{~kg}(\mathrm{SD}=0.47)$ and gestational age range of 26-32 weeks. Babies took 34.08 hrs to wean off in CPAP with KMC as compared to $38.67 \mathrm{hrs}$ in those who received only CPAP. On weaning from CPAP there was no oxygen requirement and no apnoeic episodes in both groups. Average days to reach maximum feeding were two days with no differences between groups. Intolerance of feed was a problem in the non-intervention group.

Conclusion $\mathrm{KMC}$ is feasible in babies on CPAP irrespective of weight and prematurity. It reduced the number of hours on CPAP and reduced intolerance of feeds.

\section{VALUE OF PORTABLE TRANSTHORAC ULTRASOUND TO AID ENDOTRACHEAL PLACEMENT IN EXTREMELY LOW BIRTH WEIGHT INFANTS IN THE DELIVERY ROOM}

doi:10.1136/archdischild-2012-302724.1810

'RK Philip, ${ }^{2 B}$ Kenny, ${ }^{1} \mathrm{~A}$ Ismail, ${ }^{3} \mathrm{C}$ Quinn. 'Paediatrics \& Neonatology, University Maternity Hospital Limerick; ${ }^{2}$ Radiology, University Hospital Limerick; ${ }^{3}$ Nursing \& Midwifery Neonatal Unit, University Maternity Hospital Limerick, Limerick, Ireland

Background and Aims Correct endotracheal tube (ETT) position for extremely low birth weight (ELBW) neonates during initial resuscitation is critical. We aimed to, 1 . Assess the feasibility and diagnostic utility of portable transthoracic ultrasound (PTU) to assess symmetry of diaphragmatic movement as a measure of correct ETT, 2. Assess PTU as a recordable accurate tool to document ETT position prior to surfactant administration to improve the 'golden hour management'.

Methods Single centre prospective study involving ELBW neonates $<1000$ gm requiring intubation post-delivery. Two recordings per infant were done - one in delivery room and second when the infant reaches NICU. Accuracy of PTU (Micromaxx ${ }^{\circledR}$ ) was compared with clinical assessments, colorimetric $\mathrm{CO}_{2}$ detection and Chest X-ray ETT position. Single operator conduced examinations who was not part of the resuscitation team. Hospital Research and Ethics committee approval was obtained.

Results Seventeen ELBW infants had PTU in labour delivery room ( $\mathrm{n}=17$ ) yielding 34 recordings. For 5 out of $17(29.4 \%)$ infants significant improvement of ETT position could be offered by the use of PTU which otherwise was not detected. It is feasible to measure and record diaphragmatic excursion bilaterally during the labour ward resuscitation environment. The diagnostic accuracy of PTU for correct ETT was greater than that by traditional clinical methods and colorimetric $\mathrm{CO}_{2}$ detection. Inter-operator consistency and value of hand-held device (VScan ${ }^{\circledR}$ ) is being evaluated.

Conclusion PTU is a valuable adjunct tool to record symmetry of diaphragmatic movement as a measure of correct ETT placement in labour ward for ELBW infants.

\section{HIGH-FLOW NASAL CANNULAE FOR RESPIRATORY SUPPORT OF PRETERM INFANTS: A REVIEW OF THE EVIDENCE}

doi:10.1136/archdischild-2012-302724.1811

${ }^{1} \mathrm{CC}$ Roehr, ${ }^{2,3,4 \mathrm{BJ}}$ Manley' 1 SK Dold, 2,3,4PG Davis. 'Department of Neonatology, Charité University Medical Centre, Berlin, Germany; ${ }^{2}$ Department of Newborn Research, The Royal Women's Hospital; ' ${ }^{3}$ Murdoch Childrens Research Institute; "Department of Obstetrics and Gynaecology, The University of Melbourne, Parkville, VIC, Australia

Background High-flow nasal cannulae (HFNC) are gaining in popularity as a form of non-invasive respiratory support for preterm infants in neonatal intensive care units around the world. They are proposed as an alternative to nasal continuous positive airway pressure (NCPAP) for treating respiratory distress in a variety of clinical situations, including post-extubation support, primary therapy from birth, and to 'wean' from NCPAP.

Objectives To present and discuss the available evidence for the use of HFNC in various roles in the preterm population.

Methods We performed an internet-based literature search for relevant, original research articles (both randomised and not) on the use of HFNC in preterm infants.

Results 18 studies were included in the review. Distending pressure generated by HFNC in preterm infants increases with increasing flow rate and decreasing infant size, and may vary according to the amount of leak around the prongs. HFNC may be as effective as NCPAP at improving respiratory parameters such as tidal volume and work of breathing in preterm infants, but perhaps only at flow rates $>2$ Litres per minute. Based on available published evidence, the efficacy and safety of HFNC in preterm infants remain to be determined.

Conclusions There is increasing evidence from clinical trials to support the use of HFNC treatment of preterm infants with respiratory failure, however uncertainty remains about efficacy, safety and optimal flow rates. Until the results of randomised trials in progress are known, widespread use of HFNC to treat preterm infants cannot be recommended. 